\title{
ALGORITMO DISTRIBUÍDO PARA ATRIBUIÇÃO DINÂMICA DE TAREFAS EM UM ENXAME DE ROBÔS
}

\author{
Rafael Mathias de Mendonça*, Nadia Nedjah*, Luiza de Macedo Mourelle ${ }^{\dagger}$ \\ *Departamento de Engenharia Eletrônica e Telecomunicações, Faculdade de Engenharia \\ Universidade do Estado do Rio de Janeiro \\ Rio de Janeiro, RJ, Brasil \\ ${ }^{\dagger}$ Departamento de Engenharia de Sistemas e Computação, Faculdade de Engenharia \\ Universidade do Estado do Rio de Janeiro \\ Rio de Janeiro, RJ, Brasil
}

Emails: rmathias.mendonca@gmail.com, nadia@eng.uerj.br, ldmm@eng.uerj.br

\begin{abstract}
This paper proposes a distributed control algorithm to implement dynamic task allocation in a swarm robotics environment. In this context, each robot that integrates the swarm must run the algorithm periodically in order to control the underlying actions and decisions. The algorithm was implemented and extensively tested. The corresponding performance and effectiveness are promising.
\end{abstract}

Keywords - Dynamic task allocation, Swarm robotics, Swarm intelligence, Multi-Robot Systems.

Resumo - Este artigo propõe um algoritmo de controle distribuído para a alocação dinâmica de tarefas em um ambiente de robótica de enxame. Neste contexto, cada robô que integra o enxame deve executar este algoritmo periodicamente para controlar suas ações e decisões. O algoritmo foi implementado e amplamente testado. Assim seu desempenho e eficácia são promissores.

Palavras-chave- Alocação dinâmica de tarefas, Robótica de enxame, Inteligência de enxame, Sistemas multirobôs

\section{Introdução}

A Inteligência de Enxame foi proposta no final de 1980 através da observação do comportamento social de espécies de insetos e pássaros mostrada em Bonabeau et al. (1999). Estas espécies realizam tarefas simples coletivamente com o intuito de alcançar objetivos complexos e impossíveis de serem realizados por apenas um indivíduo do enxame. A ideia central deste comportamento coletivo é a de executar uma tarefa complexa dividindo-a em tarefas simples que são facilmente executadas pelos membros do enxame. A abordagem da robótica de enxame surgiu a partir desta observação estendida a grupos de robôs móveis com capacidade de processamento limitada.

A alocação dinâmica de tarefas é um processo necessário para o bom gerenciamento do enxame. Este permite distribuir as tarefas, a serem realizadas, entre os robôs do enxame de tal maneira que uma proporção pré-definida de execução de tarefas seja respeitada. A proporção deveria ser determinada no sentido de permitir o alcance do objetivo global do enxame. A alocação de tarefas é um processo dinâmico pois precisa ser continuamente ajustada em resposta às alterações no ambiente e/ou no desempenho do enxame. Uma solução imediata para resolver este problema baseia-se na abordagem centralizada. No entanto, uma alocação distribuída representa uma melhor aproximação do comportamento dos enxames de espécies sociais, onde não existe um mecanismo de controle centralizado. Portanto, a alocação de tarefas em enxames de robôs deve surgir como resultado de um processo distribuído. Esta descentralização aumenta a complexidade do problema, pois o robô não tem uma visão completa do ambiente. Cada robô deverá tomar decisões de controle locais sem o conhecimento completo do que outros robôs fizeram no passado, estão fazendo agora ou irão fazer no futuro.

Diversas aplicações que são automatizadas usando a robótica de enxame, requerem uma alocação dinâmica de tarefas. Por exemplo, em situações que representem um risco ou inviabilizem a presença humana para a execução de uma tarefa, o enxame de robôs seria capaz de se auto-organizar para formar grupos, onde cada grupo executaria uma tarefa específica com o objetivo, de juntos realizarem uma ação mais significativa e complexa. Com a adição do elemento dinâmico de alocação de tarefas, o enxame ganharia uma maior flexibilidade e confiabilidade.

Neste artigo, é proposto um algoritmo distribuído que visa realizar a alocação dinâmica de tarefas em um enxame de robôs. O algoritmo é descentralizado e muito simples, permitindo uma execução eficiente em robôs dotados de recursos de armazenamento e processamento limitados. O algoritmo foi testado no software de prototipagem Webots PRO 6.2.1 para comprovar a sua eficácia e eficiência. Os resultados das experiências são relatados.

O restante deste artigo é organizado em 6 seções. Inicialmente, na Seção 2 é apresentado formalmente o problema de alocação dinâmica de ta- 
refas. Após, na Seção 3, são apresentados alguns trabalhos relacionados. Posteriormente, na Seção 4, são detalhados os principais passos do algoritmo proposto para resolver o problema em questão. A seguir, na Seção 5, são comentadas algumas questões de implementação sobre o software utilizado. Após, na Seção 6, são discutidos os resultados dos experimentos realizados. Por fim, na Seção 7, são comentadas algumas conclusões em relação ao algoritmo proposto e apontadas algumas direções relevantes para o trabalho futuro.

\section{Atribuição Dinâmica de Tarefas}

Normalmente, um enxame de robôs precisa realizar uma tarefa complexa que é dividida em outras tarefas menos complexas. Portanto, a atribuição dessas tarefas aos robôs no enxame é um requisito para muitas aplicações. Para realizar a atribuição da tarefa, primeiro é necessário determinar corretamente a distribuição do enxame no ambiente e em seguida distribuir as tarefas entre os robôs. Existem diversas estratégias para alocação dinâmica de tarefas. A atribuição pode ser aleatória, dependendo apenas da capacidade do robô para realizar de forma eficaz a tarefa alocada, sequencial, em paralelo ou uma combinação das duas, que permita atingir um equilíbrio entre o tempo gasto na comunicação e na execução.

De modo a proporcionar uma descrição formal do problema de atribuição dinâmica de tarefas, temos $T=\left\{t_{1}, t_{2}, \ldots, t_{\tau}\right\}$ seja um conjunto de $\tau$ tarefas a serem executadas por $\eta$ robôs do enxame. A tarefa precisa ser alocada para o robô de forma que a proporção fixada $P$ seja sempre respeitada. Seja $P=\left\{p_{1}, p_{2}, \ldots, p_{\tau}\right\}$, tal que $0 \leq p_{i} \leq 1 \mathrm{e}$ $p_{1}+p_{2}+\cdots+p_{\tau}=1$. Para satisfazer a proporção de tarefas imposta, nós precisamos, para todas as tarefas $t \in T$, satisfazer $S_{t}=p_{t} \times \eta$, onde $S_{t} \leq \eta$ é o número total de robôs atribuídos à tarefa $t$.

\section{Trabalhos relacionados}

A análise do comportamento coletivo em Sistemas Multi-Robô é um campo de estudo relativamente novo que abrange metodologias da Matemática, Física e Biologia. Os primeiros estudos relevantes nesta área com a expressiva intenção de descentralizar o controle no processo de alocação de tarefas são apresentados em Sugawara and Sano (1997) e Sugawara et al. (1998). Nestes trabalhos foram realizados estudos com grupos mistos de robôs divididos em robôs que possuíam algum tipo de comunicação centralizada e robôs que baseavam suas decisões apenas em observações locais. Em Kazadi et al. (2002) foram estudadas as propriedades gerais de agregação em Sistemas Multi-Robô usando modelos fenomenológicos macroscópicos.

Novas formulações foram desenvolvidas para a criação de modelos fenomenológicos direciona- das para um comportamento coletivo de grupos de robôs em Lerman and Galstyan (2004) e Lerman et al. (2005). Estas novas formulações foram experimentadas em testes colaborativos simples como em Lerman et al. (2001) onde foi realizado o experimento stick-pulling em um grupo de robôs reativos.

A maior parte das abordagens supra-citadas são implícita ou explicitamente baseadas na teoria de processos estocásticos. O modelo Probabilístico Microscópico desenvolvido em (Martinoli (1999), Martinoli et al. (1999) e Ijspeert et al. (2001)) é um exemplo da abordagem estocástica para estudar o comportamento coletivo de um grupo de robôs. O impacto de vários eventos estocásticos em paralelo, para cada robô do enxame, permitiu a elaboração de algumas propriedades sobre o comportamento dos robôs em Sistemas Multi-Robô.

\section{Algoritmo Proposto}

O algoritmo de alocação de tarefas proposto é gerenciado periodicamente pelos $\eta$ robôs presentes no enxame. Note que as execuções do algoritmo ocorrerão simultaneamente, sendo uma em cada robô, conforme mostrado no Algoritmo 1, onde $t_{i}$ representa o identificador da tarefa alocada atualmente ao robô $i$. Sendo a o endereço deste robô e $T_{i}$ o conjunto de identificadores das tarefas alocadas aos demais robôs, excluindo o próprio robô $i$. Assim, o processo de ajuste, denominado AjustarTarefa, que permite selecionar a tarefa a ser realizada é executado por cada um dos robôs, conforme percebido por este robô.O robô pode escolher entre uma das $\tau$ tarefas disponíveis. 
da proporção imposta. Isto é feito através de vários ajustes de tarefas, chamada AjustarTarefa. Quando a tarefa para o robô é ajustada, este entra na etapa de execução. Durante esta etapa, o robô executa a tarefa ajustada, através de ExecutarTarefa. O mesmo processo é iterado. Note que ao executar a tarefa que lhe foi atribuída, o robô se comunica com os robôs do enxame enviando a sua tarefa e recebendo as tarefas dos demais robôs para verificar o cumprimento da proporção imposta.

Seja $t_{i}$ a tarefa alocada ao robô $i$. O Algoritmo 2 descreve o método proposto para operacionalizar o ajuste de tarefas alocadas aos robôs do enxame. Neste algoritmo, $P$ representa a proporção desejada de robôs executando cada uma das $\tau$ tarefas. 


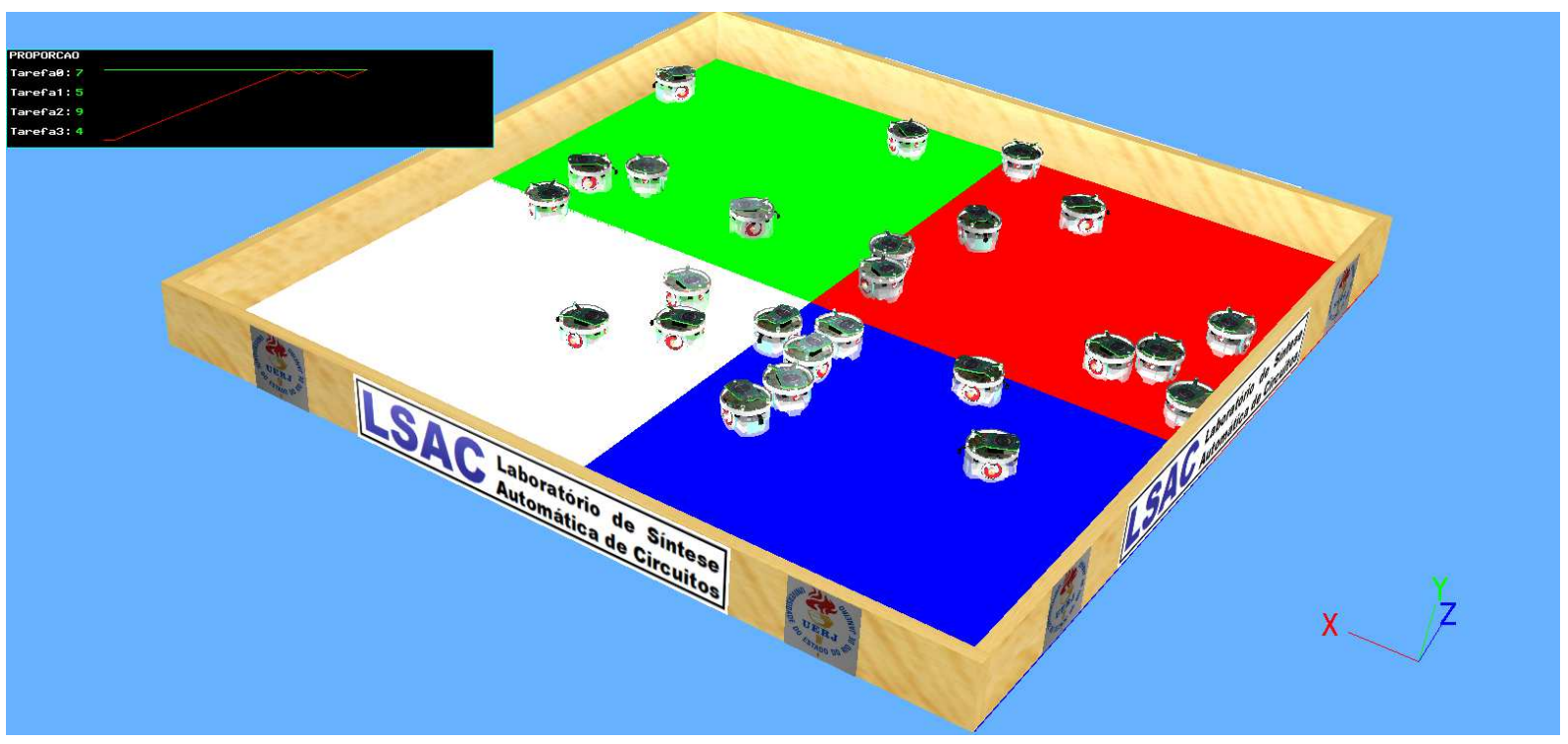

Figura 2: Exemplo de aplicação do algoritmo proposto

\section{Performance dos Resultados}

Como um exemplo de aplicação para o algoritmo, propomos a realização de uma simulação capaz de representar um comportamento coletivo através da execução de 4 simples tarefas pelos robôs do enxame. Nesta, um enxame de 25 robôs é iniciado realizando a tarefa 0 e no decorrer do tempo as tarefas são dinamicamente alocadas com o objetivo de convergir para uma proporção pré-definida de 7, 5, 9 e 4 robôs executando as tarefas $0,1,2$ e 3 , respectivamente. Cada tarefa é representada por uma cor (Azul - Tarefa 0; Verde - Tarefa 1; Vermelho - Tarefa 2; Branco - Tarefa 3). Os robôs andam aleatoriamente pelo ambiente desviando dos obstáculos usando seus sensores IR e visualizando o ambiente através de uma câmera VGA direcionada para o chão. Sempre que a câmera focaliza uma imagem que corresponde a cor designada pela tarefa que está sendo realizada por este robô neste momento este paralisa seu deslocamento e permanece imóvel enquanto esta situação for verdadeira.

O resultado final é mostrado na Fig. 2, onde o enxame foi dividido entre os quatro quadrantes do ambiente exatamente na proporção definida inicialmente. Assim teremos os 7 robôs realizando a tarefa 0 no quadrante Azul, os 5 robôs realizando a tarefa 1 no quadrante Verde, os 9 robôs realizando a tarefa 2 no quadrante Vermelho e os 4 robôs restantes realizando a tarefa 3 no quadrante Branco. Um vídeo desta simulação esta disponível em http://www. youtube.com/watch? $\mathrm{v}=\mathrm{bVmuWM} 4 \mathrm{et} 8 \mathrm{w}$.

Com o objetivo analisar a performance do algoritmo proposto em relação ao tempo de execução e de comunicação, foram realizados vários testes. Nós variamos a configuração do enxame em termos do número de robôs, bem como o número de tarefas distintas que estes devem realizar res-
Tabela 1: Distribuição da proporção entre tarefas 


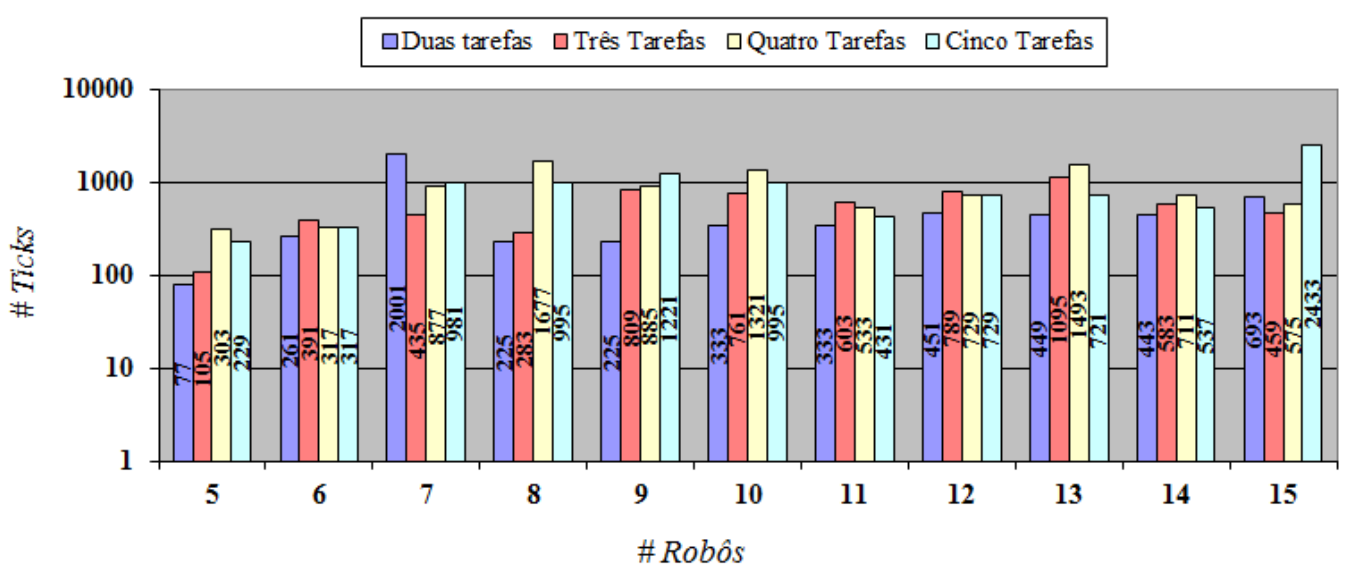

Figura 3: Tempo de execução em ticks de sistema durante a realização dos testes

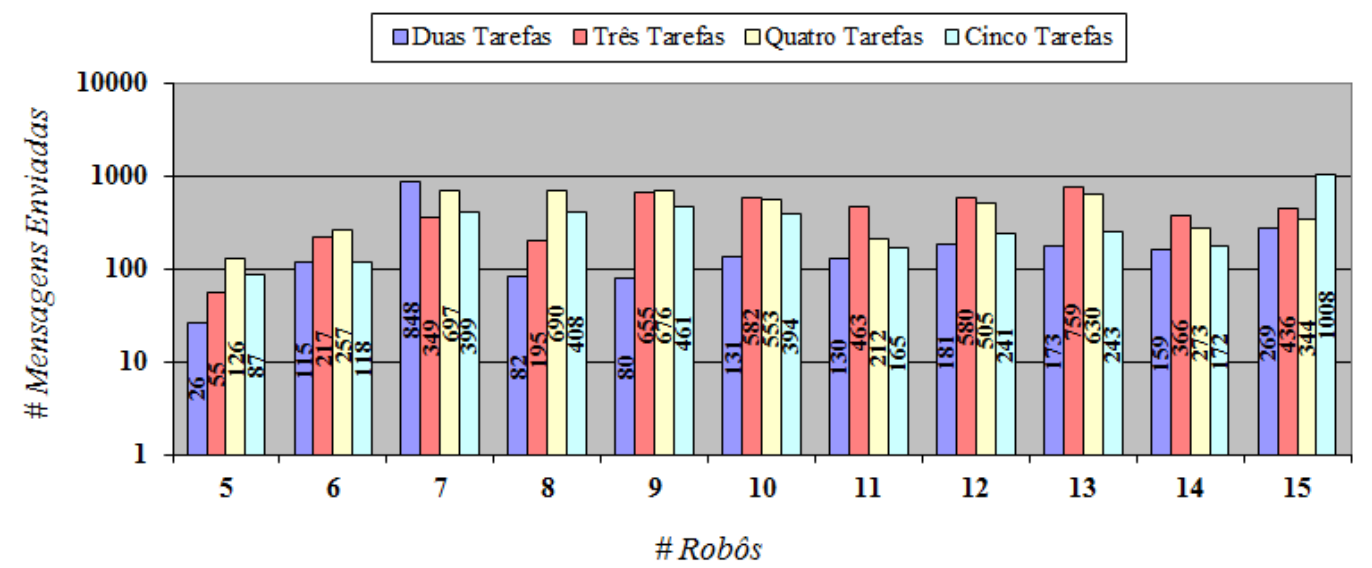

Figura 4: Número de mensagens enviadas durante a realização dos testes

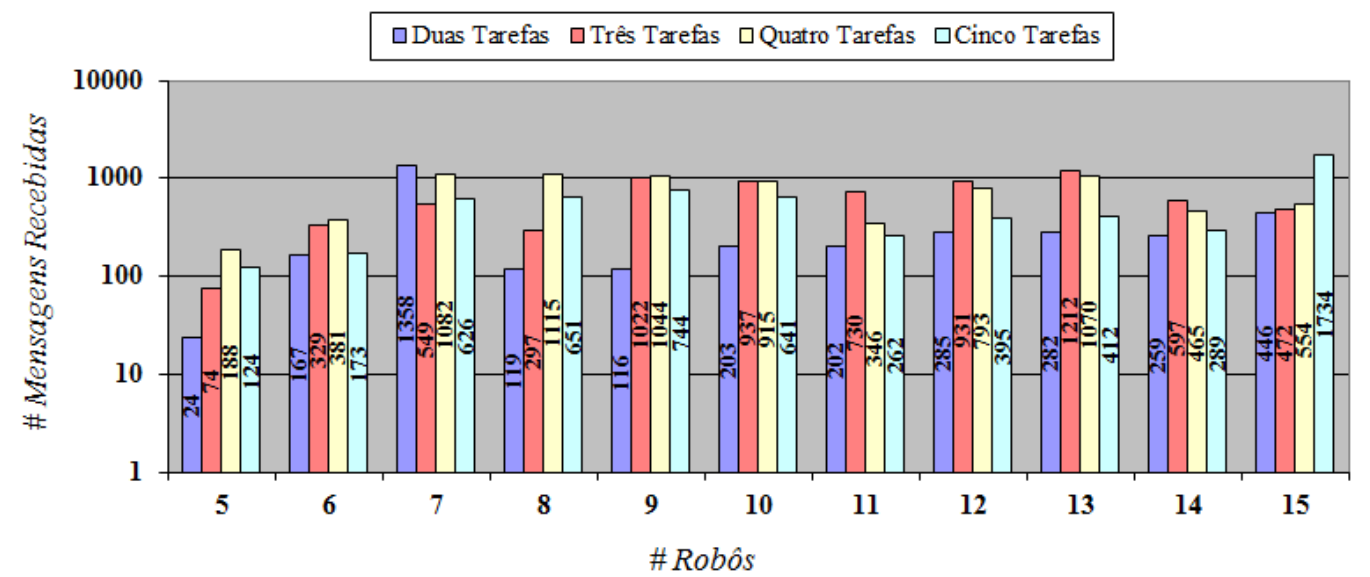

Figura 5: Número de mensagens recebidas durante a realização dos testes

viada e recebida pela entidade supervisora, bem como o número de ticks decorridos. Quando a proporção prescrita é alcançada, conforme percebida pelo gerenciamento central na figura da entidade supervisora, o estado dos três contadores está registado. Note que a distribuição de tarefas pode oscilar em torno da proporção prescrita antes de se tornar estável.

Observamos que o número de ticks do sistema e as mensagens enviadas e recebidas pela entidade supervisora aumenta com o número de robôs no enxame na maioria dos casos. Podemos observar também que o número de tarefas utilizadas tem uma influência direta sobre a complexidade da decisão e do processo de atribuição de tarefas, resultando em um aumento em termos de mensagens enviadas e recebidas, e portanto, também do número de ticks. 


\section{Conclusão}

Neste artigo, propomos o uso de um algoritmo descentralizado para gerenciar a atribuição de tarefas entre um enxame de robôs, a fim de atingir e manter a proporção pré-definida. O algoritmo proposto é uma solução eficiente para a atribuição de tarefas em um enxame de robôs. O processo de decisão é feito de forma independente por cada robô através de um processo simples e direto. Os resultados dos testes mostram o tempo de execução do algoritmo proposto, bem como as estatísticas das mensagens geradas pelo processo de comunicação. Este algoritmo foi implementado em robôs móveis reais do tipo Elisa3, como mostrado em de Mendonça et al. (2013), apresentando resultados coerentes com os resultados observados na simulação.

Em um trabalho futuro, pretende-se investigar o impacto de diferentes proporções, a fim de avaliar a influência dessas proporções no processo de convergência.

\section{Agradecimentos}

Somos gratos a FAPERJ (Fundação de Amparo à Pesquisa do Estado do Rio de Janeiro, http://www.faperj.br) e CNPq (Conselho $\mathrm{Na}$ cional de Desenvolvimento Científico e Tecnológico, http://www. cnpq.br) a CAPES (Coordenação de Aperfeiçoamento de Pessoal de Ensino Superior, http://www. capes .gov.br) por seu contínuo apoio financeiro.

\section{Referências}

Bonabeau, E., Dorigo, M. and Theraulaz, G. (1999). Swarm intelligence: from natural to artificial systems, number 1, Oxford University Press, USA.

de Mendonça, R. M., Nedjah, N. and de Macedo Mourelle, L. (2013). Efficient distributed algorithm of dynamic task assignment for swarm robotics, Computational Science and Its Applications-ICCSA 2013, Vol. 7971, Springer.

Ijspeert, A., Martinoli, A., Billard, A. and Gambardella, L. (2001). Collaboration through the exploitation of local interactions in autonomous collective robotics: The stick pulling experiment, Autonomous Robots 11(2): 149171. DOI: $10.1023 / \mathrm{A}: 1011227210047$

Kazadi, S., Abdul-Khaliq, A. and Goodman, R. (2002). On the convergence of puck clustering systems, Robotics and Autonomous Systems 38(2): 93-117. DOI: 10.1016/S0921-8890(01)00171-3
Lerman, K. and Galstyan, A. (2004). Collectives and Design of Complex Systems, Springer Verlag, New York, chapter Two Paradigms for the Design of Artificial Collectives, pp. 231-256.

Lerman, K., Galstyan, A., Martinoli, A. and Ijspeert, A. (2001). A macroscopic analytical model of collaboration in distributed robotic systems, Artificial Life 7(4): 375-393.

Lerman, K., Martinoli, A. and Galstyan, A. (2005). A review of probabilistic macroscopic models for swarm robotic systems, Swarm Robotics pp. 143-152.

Martinoli, A. (1999). Swarm intelligence in autonomous collective robotics: From tools to the analysis and synthesis of distributed control strategies, $\mathrm{PhD}$ thesis.

Martinoli, A., Ijspeert, A. and Gambardella, L. (1999). A probabilistic model for understanding and comparing collective aggregation mechanisms, Advances in Artificial Life pp. $575-584$.

Sugawara, K. and Sano, M. (1997). Cooperative acceleration of task performance: Foraging behavior of interacting multi-robots system, Physica D: Nonlinear Phenomena 100(3-4): 343-354.

Sugawara, K., Yoshihara, I., Abe, K. and Sano, M. (1998). Cooperative behavior of interacting robots, Artificial life and Robotics 2(2): 6267. 